\title{
CONSTRUCCIONES DE PARTICIPIO Y DE INFINITIVO EN TEXTOS PORTUGUESES MEDIEVALES Y RENACENTISTAS
}

\section{Introducción}

El objetivo de este estudio es examinar el orden de palabras en las construcciones constituidas por un verbo regente y un participio o un infinitivo dependiente en textos portugueses medievales y prerrenacentistas, teniendo en cuenta principalmente la posibilidad de la anteposición de las formas verbales dependientes y la separabilidad de los dos verbos que componen cada una de estas construcciones. Los ejemplos que se aducen en (1-2) ilustran los dos fenómenos, habituales en el portugués antiguo, pero agramaticales o por lo menos muy restringidos en las variedades del portugués actual.

(1) a. Departido avemos em que maneira reynou este rey dom Ramiro

(CroGer, 114a)

b. Enganados fomos noutro dia (Demanda, 7d)

c. Mas os outros mouros que fogir poderon acolheronsse a hũũ castello que (Cge, 115b)

d. Porem ainda que pareça trabalhoso aprender e custumarsse aas ditas partes do entendimento, todavia custumalas devemos (LeaCon, 5v)

(2) a. quando o conde esto dezia, o sol era ja posto (CroGer, 125b)

b. ca hoje sera'a minha corte chamada por sempre Corte Aventurosa (Demanda, 5a)

c. depois que ouve os seus todos ajuntados, (CroGer, 118b)

d. $\quad$ E se nõ quiserẽ end(e) auijr os omees boos que recebã proua da suspeyta (ForRea, 78)

e. $\quad$ E entõ ouverõ todollo seus de ajuntar ally onde estava a sua syna (CroGer, 125b)

f. que os nõ poderyã melhores achar em nẽũ hũa parte (CroGer, 124a)

Dichos fenómenos, presentes también en español, fueron examinados en las construcciones de participio del español antiguo por Company ${ }^{1}$, Andres-Suárez ${ }^{2}$ y García Martín ${ }^{3}$. Los estudios clásicos de gramática histórica del portugués, ya

\footnotetext{
${ }^{1}$ Concepción COMPANY, "Sintaxis y valores de los tiempos compuestos en el español medieval”, in: Nueva Revista de Filología Hispánica, XXXII/2, 1983, págs. 235-257.

${ }^{2}$ Irene ANDRES-SUÁREZ, El verbo español. Sistemas medievales y sistema clásico. Madrid, Gredos, 1994.

3 José María GARCÍA MARTÍN, La formación de los tiempos compuestos del verbo en español medieval y clásico. Aspectos fonológicos, morfológicos y sintácticos. Valencia, Universitat de València, 2001.
} 
anticuados, como el de Huber ${ }^{4}$ y el de Dias ${ }^{5}$ ofrecen muy pocas informaciones sobre las cuestiones del orden de palabras. En mis trabajos dedicados a la estructura de las construcciones de infinitivo aparece el aspecto comparativo entre el español y el portugués $^{6}$, pero dejando de lado la comparación con las construcciones de participio. En este trabajo me concentraré, pues, en el estudio de la evolución del orden de palabras tanto en las construcciones de participio como en las construcciones de infinitivo, examinando, de un lado, el fenómeno de la anteposición de los verbos no flexionados dependientes y, de otro, la interpolación de constituyentes entre el verbo regente y el verbo complemento. El punto de partida del análisis es la hipótesis que supone que la anteposición de los verbos complementos, así como la interpolación, eran posibles en la variante medieval de todas las lenguas romances peninsulares gracias al funcionamiento del sistema $\mathrm{V} 2$, que colocaba el verbo en la segunda posición de la frase y permitía que cualquier constituyente ocupase la primera. La sustitución de este sistema por el nuevo orden SVO causaría que el participio y el infinitivo -como complemento regido por el verbo regente- se situasen a la derecha de éste y que los demás complementos desapareciesen de las posiciones intermedias. La consecuencia de estos cambios sería, pues, que se fijasen como órdenes habituales $\operatorname{VPart}(\mathrm{O})$ y $\operatorname{VInf}(\mathrm{O})$ en la variedad actual del portugués ${ }^{7}$.

Actualmente tenemos suficientes conocimientos acerca de la naturaleza de los cambios lingüísticos para pensar que la anteposición y la interpolación, rasgos característicos de la lengua medieval, no habrán desaparecido de repente, sino gradualmente en un período de transición entre la fase medieval y la fase actual de la historia de la lengua. También es de suponer, aunque no se dispone de referencias tan concretas como en el caso del español al respecto, que las estructuras antiguas pueden surgir en el portugués actual, sea por arcaísmo de algún autor sea por las características de algún tipo de texto. Incluso es lógico conjeturar -como hicimos durante el análisis de los mismos fenómenos en español- que la anteposición y la interpolación, que en la Edad Media eran solamente alternativas de otras posibilidades sintácticas, originariamente no se usarían con la misma frecuencia en los distintos tipos de textos, y que su retroceso tampoco ocurriría necesariamente con la misma rapidez en los diferentes géneros o estilos literarios. Para comprobar la validez de esta hipótesis se realizó el análisis de la frecuencia estadística de la anteposición del participio y del infinitivo en textos portugueses medievales procedentes del período que abarca los siglos entre XIII y XV. Los objetivos eran idénticos a los que se habían formulado en las investigaciones referentes al español:

4 J. HUBER, Altportugiesisches Elementarbuch, Heidelberg, Carl Winters Universtitätbuchhandlung, 1933. En portugués: J. HUBER, Gramática do português antigo, Lisboa, Fundação Calouste Gulbenkian, 1986.

5 A. E. da Silva DIAS, Syntaxe Histórica Portuguesa, Lisboa, Livraria Clásica, 1970 (primera edición: 1917).

${ }^{6}$ Tibor BERTA, "La estructura de las construcciones de infinitivo en textos españoles y portugueses medievales", in: Sándor KISS- Giampaolo SALVI-Ildikó SZIJJ (eds.), rom.hu. Études Romanes de Budapest, 3. Budapest, Programme Doctoral de Linguistique Romane de l'Université Eötvös Loránd/Íbisz, 2001, 33-47. Idem, Clíticos e infinitivo. Contribución a la historia de la promoción de clíticos en español y portugués. Szeged, Hispánia, 2003.

${ }^{7} \mathrm{~V}$ equivale a verbo regente, Part a participio, Inf a infinitivo y $(O)$ representa la posición de los complementos opcionales en la frase. 
a) comparación de las construcciones de participio con las de infinitivo;

b) comparación sincrónica de diferentes tipos de textos de la misma época;

c) comparación de textos del mismo tipo procedentes de épocas distintas.

Para alcanzar estos objetivos se creó un corpus constituido por textos portugueses redactados entre el siglo XIII y el siglo XV, que pertenecen a los siguientes tipos: textos jurídicos, crónicas y obras literarias. Se ha excluido la poesía teniendo en cuenta los factores de la versificación que permiten una mayor libertad sintáctica al poeta de la que existe en el habla realmente. El Cuadro 3.1. presenta los datos más importantes del corpus seleccionado ${ }^{8}$. En la columna "Palabras" aparece la extensión de cada texto o fragmento examinado; en la columna "Inf” figura el número de aquellas construcciones registradas que contienen un verbo regente y un infinitivo dependiente; las cifras bajo "Part" se refieren a las construcciones de participio que contienen un verbo regente cuyo complemento es un participio; la columna "Total" indica el número de todos los ejemplos registrados en un texto analizado. Nótese que se han excluido los participios absolutos y las construcciones con infinitivo de función adverbial, porque en estos casos el participio y el infinitivo no dependen estrictamente del verbo flexionado.

\begin{tabular}{|l|r|r|r|r|}
\hline Textos portugueses & Palabras & \multicolumn{1}{c|}{ Inf } & \multicolumn{1}{c|}{ Part } & \multicolumn{1}{c|}{ Total } \\
\hline CroGer & 15.000 & 321 & 198 & 519 \\
\hline ForRea & 12.300 & 94 & 75 & 169 \\
\hline Demanda & 9.000 & 156 & 58 & 214 \\
\hline Zurara & 10.000 & 95 & 35 & 130 \\
\hline CasPer & 7.600 & 179 & 58 & 237 \\
\hline LeaCon & 5.000 & 90 & 43 & 133 \\
\hline Total portugueses & 58.900 & 935 & 467 & 1.402 \\
\hline
\end{tabular}

Cuadro 3.1.

Constitución del corpus portugués

\footnotetext{
${ }^{8}$ La mayor parte de los textos portugueses analizados procede del Corpus Informatizado do Português Medieval (CIPM), accesible a través de la siguiente página web, mantenida por un grupo de investigación del Centro de Lingüística de la Universidade Nova de Lisboa: http://cipm.fcsh.unl.pt; las referencias bibliográficas de las fuentes informatizadas son las siguientes: ForRea=Foro Real [1280?], a partir de José de AZEVEDO (ed.), Foro Real, Lisboa, INIC; CroGer= Crónica Geral de Espanha de 1344 [sin fecha, manuscrito del siglo XIV], a partir de Luís Filipe Lindley CINTRA (ed.), Crónica Geral de Espanha de 1344, Lisboa, INCM, 1951; Demanda=A Demanda do Santo Graal, Códice 2594 da Bibiloteca Nacional de Viena [manuscrito del siglo XV]; CasPer=Castelo Perigoso [sin fecha, manuscrito del siglo XV], João António Santana NETO (ed.), Duas Leituras do Tratado Ascético-Místico Castelo Perigoso, Dissertação de Doutoramento, São Paulo, Faculdade de Filosofia, Letras e Ciências Humanas, USP, 1997, edição revista por Irene Nunes; LeaCon=Dom DUARTE, Leal Conselheiro, a partir de Joseph PIEL (ed. crít.), Leal Conselheiro, Lisboa, Livraria Bertrand, edição digitalizada, revista por João Dionísio e Sandra Álvarez. Además de estos textos informatizados se utilizó la versión impresa de la siguiente obra: Zurara=Gomes Eanes de ZURARA: Prosas históricas, ed. de RODRIGES LAPA, Lisboa (sin año de edición).
} 


\section{Proporción de la anteposición}

El primer fenómeno analizado ha sido el de la anteposición del verbo en forma de participio o infinitivo, ya ilustrado en los estudios precedentes de este tomo. Tal fenómeno se documenta también en los textos portugueses analizados con ambos tipos de construcciones. El Cuadro 3.2. resume los datos referentes a la proporción de la anteposición del participio al verbo regente, el cual puede ser ser, estar, haver, ter. Nótese que desde el punto de vista del fenómeno es indiferente si se trata de construcción perifrástica o no, o si el significado de la construcción es pasivo o no.

\begin{tabular}{|l|r|r|r|}
\hline \multicolumn{1}{|c|}{ Textos } & \multicolumn{1}{c|}{ VPart } & \multicolumn{1}{|c|}{ PartV } & \multicolumn{1}{c|}{ Total } \\
\hline ForRea & 73 & 2 & 75 \\
\hline CroGer & 191 & 7 & 198 \\
\hline Demanda & 51 & 7 & 58 \\
\hline Zurara & 35 & 0 & 35 \\
\hline CasPer & 57 & 1 & 58 \\
\hline LeaCon & 41 & 2 & 43 \\
\hline Total & 448 & 19 & 467 \\
\hline \multicolumn{4}{|c|}{ Cuadro 3.2. } \\
\hline
\end{tabular}

Anteposición del participio en portugués

Como se puede ver, se han recogido en total 19 casos con participio antepuesto al verbo regente; esta cantidad de ocurrencias constituye el $4 \%$ del total de los casos de construcciones de participio. Al comparar los textos anteriores al siglo XV con los de este mismo siglo observamos una diferencia considerable en la frecuencia del participio antepuesto: en los tres textos medievales la anteposición alcanza el 4,8\% -16 casos de 331 construcciones-, mientras que en los renacentistas llega al 2,2\% -3 casos de 136 construcciones-. En cuanto a la proporción del fenómeno según textos, el porcentaje más alto lo encontramos en dos textos medievales, en Demanda - $12 \%$ - y en CroGer $-3,5 \%-y$, además, en LeaCon -4,6\%-. En los demás textos la proporción del fenómeno se encuentra alrededor del $2 \%$, excepto Zurara, en el que no se ha registrado ningún caso de anteposición.

Las ocurrencias con participio antepuesto recogidas en el corpus son las que se presentan en (3-7).

(3) a. Departido avemos em que maneira reynou este rey dom Ramiro (CroGer, 114a)

b. ẽviou o conde algũũs de seus ricos homẽẽs que lhe dissessem que elle aparelhado estava pera seu serviço e nõ pera seu deserviço (CroGer, 116d)

c. de non consentirem, a nehũũ senhor que ouvessem, que fezesse nen hũa cousa que desaguisada nem sem razon fosse (CroGer, 118c)

d. E Almãçor hya dizendo que irado avya naquelle dya Mafomede (CroGer, 120a)

e. ca elles vencidos e desbaratados som. (CroGer, 126a) como dicto he (CroGer, 121b)

f. ca vençudos som (CroGer, 126b) 
g. nũca tanto esforçados foram em nẽ hũũ dos outros dyas dante este (CroGer, 125d)

(4) a. se algũas cousas das eygreyas uendudas acharẽ ou alleadas ou mal baratadas sen dereyto (ForRea, 74v)

b. $\quad$ assy como ya dito e' (ForRea, $77 \mathrm{v})$

(5) a. E metuda hei minha esperança em Nosso Senhor (Demanda, 1d)

b. morto som (Demanda, 3b)

c. Pois feito e' (Demanda, 4b)

d. ja' tam ardido nom seria (Demanda, 7a)

e. $\quad$ Enganados fomos noutro dia (Demanda, 7d)

f. beento seja o dia em que vo's nascestes (Demanda, 7d)

g. $\quad$ cousa que lhes outorgada fosse por afam (Demanda, 8c)

(6)

Assi como dicto he que (CasPer, 9v)

(7) a. Ca scripto he: ... (LeaCon, 3r)

b. E por quanto esto screvo, como dito he, por comprir vossa voontade com meu prazer e desenfadamento (LeaCon, $4 \mathrm{r}$ )

Entre las construcciones de infinitivo se han registrado 11 casos en los que el infinitivo precede al verbo regente; esta cantidad de ocurrencias constituye el 1,2\% de las 935 construcciones de infinitivo. La anteposición se distribuye de la siguiente manera entre los textos medievales y renacentistas: en los primeros alcanza el 1,6\% -9 casos de 571 construcciones-, en los últimos el 0,5\% -2 de 364 construcciones-. Entre los textos medievales ForRea es el único en el que no se ha registrado ningún caso de anteposición, mientras que entre los renacentistas en Zurara y CasPer tampoco. En los otros tres textos la frecuencia de la anteposición es la siguiente: el 1,2\% en CroGer, el $3,2 \%$ en Demanda y el 2,2\% en LeaCon. Estos datos se pueden observar en Cuadro 3.3.

\begin{tabular}{|l|r|r|r|}
\hline \multicolumn{1}{|c|}{ Textos } & \multicolumn{1}{c|}{ VInf } & \multicolumn{1}{c|}{ InfV } & \multicolumn{1}{c|}{ Total } \\
\hline ForRea & 94 & 0 & 94 \\
\hline CroGer & 317 & 4 & 321 \\
\hline Demanda & 151 & 5 & 156 \\
\hline Zurara & 95 & 0 & 95 \\
\hline CasPer & 179 & 0 & 179 \\
\hline LeaCon & 88 & 2 & 90 \\
\hline Total & 924 & 11 & 935 \\
\hline \multicolumn{4}{|c|}{ Cuadro 3.3. } \\
\hline
\end{tabular}

Anteposición del infinitivo en portugués

Las ocurrencias con infinitivo antepuesto al verbo regente se pueden observar en $(8-10)$ 
(8) a. Mas os outros mouros que fogir poderon acolheronsse a hũũ castello que (CroGer, 115b)

b. disselhe que, quanto fazer podesse pera partir esta lide, que o fezesse, (CroGer, 118b)

c. que lhe façã no corpo a mais crua e deshonrrada justiça que seer poder (CroGer, 123d)

d. $\quad$ se nõ todo dampno e todo mal que nos fazer poderẽ, come nossos ẽmiigos (CroGer, 123b)

(9) a. Leixar-nos queredes a atal festa (Demanda, 1b)

b. nom me semelha que comer possades (Demanda, 3a)

c. A provar vos conve'm (Demanda, 3d)

d. ca de mover havera'm ora todos aqueles que (Demanda, 6b)

e. por trabalho que sofrer pudessem (Demanda, 8c)

(10) a. Porem ainda que pareça trabalhoso aprender e custumarsse aas ditas partes do entendimento, todavia custumalas devemos (LeaCon, $5 \mathrm{v}$ )

b. o que fazer nom pode por seer batalha que nosso senhor deos nos ordenou por nosso proveito (LeaCon, 7r)

\section{Intercalación de constituyentes en portugués}

En los textos portugueses analizados -así como en los textos españoles examinados anteriormente (véase el estudio correspondiente de este mismo tomo)- se documenta la interpolación de diferentes constituyentes entre el verbo regente flexionado y el participio o el infinitivo dependiente. El Cuadro 3.4. presenta los datos referentes a la intercalación de constituyentes entre el verbo regente y el participio 9 .

\begin{tabular}{|l|r|r|r|r|r|r|}
\hline \multicolumn{1}{|c|}{ Textos } & \multicolumn{4}{|c|}{ Int+ } & Int- & Total \\
\hline & Sujeto & Objeto & \multicolumn{1}{c|}{ Adverbio } & Int+ total & & \\
\hline ForRea & 0 & 0 & 2 & 2 & 73 & 75 \\
\hline CroGer & 5 & 7 & 23 & 35 & 163 & 198 \\
\hline Demanda & 5 & 3 & 5 & 13 & 45 & 58 \\
\hline Zurara & 1 & 0 & 6 & 7 & 28 & 35 \\
\hline CasPer & 0 & 2 & 4 & 6 & 52 & 58 \\
\hline LeaCon & 0 & 2 & 6 & 8 & 35 & 43 \\
\hline \multicolumn{1}{|c|}{ Total } & 11 & 14 & \multicolumn{7}{|c|}{ Cuadro 3.4 } & 71 & 396 & 467 \\
\hline
\end{tabular}

Interpolación con participio en portugués

\footnotetext{
${ }^{9}$ Así como en los demás estudios de este tomo las abreviaturas de los cuadros referentes a la interpolación tienen los significados siguientes: Int+=con interpolación; Int-=sin interpolación; Sujeto=sujeto interpolado; Objeto=complementos regidos interpolados; Adverbio=elementos adverbiales interpolados.
} 
En los textos analizados se han recogido en total 71 construcciones en las que el verbo regente -por lo general haver o ser-está separado del participio dependiente que lo sigue. Esta cantidad de ocurrencias constituye el 15,2\% de las 467 construcciones de participio.

Si analizamos los datos de cada uno de los textos del corpus, encontramos resultados bastante diferentes. La proporción de la interpolación de constituyentes alcanza los siguientes porcentajes en los textos: ForRea: 2,6\%, CroGer: 17,7\%, Demanda: 22,4\%, Zurara: $20 \%$, CasPer: $10 \%$, LeaCon: $18,6 \%$. Esto es, sólo el texto legal presenta un porcentaje excesivamente bajo del fenómeno, en los demás encontramos una frecuencia cercana al $20 \%$, independientemente de la época de origen.

Según la categoría del constituyente interpolado destaca la proporción de los elementos adverbiales: éstos aparecen entre los dos verbos 46 veces, es decir, en el $9,8 \%$ del total de las construcciones de participio recogidas. El sujeto se interpola 11 veces, es decir, en el 2,35\%, mientras que algún argumento regido 14 veces, o sea, casi en el 3\% de los 467 casos. Los casos de interpolación con elementos adverbiales se presentan en (11-16), los casos con sujeto interpolado en (17-19), mientras que los casos con argumento regido en (20-23).

(11) a. e nõ foram hi chamados. (CroGer, 114c)

b. E Abenhya foy hy preso. (CroGer, 115b)

c. Mas el rei dom Ordonho era muy atrevudo em armas e (CroGer, 116c)

d. E o cavalleiro era muy preçado (CroGer, 117d)

e. como muy bõõ cavalleiro que elle era e cõ muy bõõs vassallos e muy leaaes que elle sempre ouve e de que foy sempre muy ben servido (CroGer, 117d)

f. foy muy sanhudo (CroGer, 118a)

g. que os mouros ficavã muito spantados dos grãdes feitos que o conde e os seus faziã (CroGer, 120a)

h. E elle estava mui mal ferydo dhũa lançada no costado destro (CroGer, 121a)

i. $\quad$ E nõ no achey, ca era ja morto (CroGer, 123a)

j. $\quad$ que achey ja morto (CroGer, 123b)

k. $\quad$ os escudeyros hyã muy bẽ armados (CroGer, 124a)

1. que ficarom muy quebrantados das feridas (CroGer, 124d)

m. quando o conde esto dezia, o sol era ja posto (CroGer, 125b)

n. E o conde e todallas suas companhas forõ aquella noyte muy ben albergados dequello que os mouros tiinham (CroGer, 125b)

o. $\quad$ E o conde e os seus de grande manh[ãã] avyam ja ouvydo missa. (CroGer, 125b)

p. que hiriã mais esforçados (CroGer, 125d)

q. por hyrem mais folgados (CroGer, 125d)

r. E muytos bõõs cavalleyros de Castella foram ally mortos em aquella lide (CroGer, 126a)

s. que era muy maravilhado (CroGer, 126d)

t. ca todollos outros de sua terra erã ja jũtados (CroGer, 127a)

u. $\quad$ como viinham muy canssados (CroGer, 127a)

v. de que elle era muy pagado (CroGer, 127b)

w. E o conde foy logo metido em grandes ferros (CroGer, 128c) 
(12) a. E as sentenças que os $\mathrm{p}(\mathrm{re})$ lados e os clerigos poserẽ sub(re) estas cousas seyã ben teudas ata que (ForRea, 76r)

b. a sentença seya logo tollecta (ForRea, 76r)

(13) a. E andava tam bem vestido que (Demanda, 1c)

b. assi como o conto ha' ja' devisado (Demanda, 3c)

c. assi nom seredes pois culpado (Demanda, 3d)

d. assi como o conto ha' ja' devisado (Demanda, 4b)

e. quero que em aquel [ca]mpo de Camaalot seja ora começado trebelho tal que (Demanda, 7a)

(14) a. andava mui anojado (CasPer, 10r)

b. que homem nom esta' bem confessado e rrepreendido (CasPer, 14r)

c. que estas duas creaturas, que tam a'sp(er)a vida faziam, p(er)derom todo $\mathrm{p}(\mathrm{er}) \mathrm{v}(\mathrm{er})$ gonha, aquelas que tantas boas obras fezerom $\mathrm{p}(\mathrm{er})$ que mereciom seer santas em parai'so, se forom bem confesadas. (CasPer, 3v)

d. foi $\mathrm{p}(\mathrm{er}) \mathrm{a}$ semp(re) condanada $\mathrm{p}(\mathrm{er})$ hũu soo conssintimento, (CasPer, 3r)

(15) a. porque fora todo mui bem tratado e encaminhado (Zurara, 2)

b. $\quad$ Ca el-rei e todos aqueles que algo seguiram nas primeiras guerras sam já cansados e enfadados (Zurara, 7)

c. tanto eles teveram mais usado o ofício das armas (Zurara, 7)

d. A gente da frota, que no começo da noute fora trabalhada, uũs em corregimento das suas fardagens, outros aparelhando as guanições de seus navios, eram ainda alguũ pouco assonorentados (Zurara, 23)

e. $\quad$ Bem os mouros nom estavam tanto ouciosos (Zurara, 25)

f. Parecendo todo armado (Zurara, 27)

(16) a. conssiirey que seria melhor feicto em forma de hũũ soo tractado com algũũs adimentos. (LeaCon, 3r)

b. que per os sabedores he muyto louvada por trazer os que se per ella regem com a graça de deos a todo bem (LeaCon, 7v)

c. com tal receo de mingua e fallecimento nom se ocupando em outras cousas que torvem o cuidado ou deligente obra, dando sem tardança devida execuçom no que ouver bem penssado. (LeaCon, 4v)

d. Compre, pera sse melhor entender, de se leer todo de começo, passo, e pouco de cada hũa vez, bem apontado, estando em razoado tempo bem despostos os que leerem e ouvirem. (LeaCon, 3v)

e. e os que hũas bestas nom enfream as outras som em elles bem aderençadas (LeaCon, 4r)

f. e as hidades som per muytas maneiras repartidas (LeaCon, 5)

(17) a. e ally foy o doo renovado (CroGer, 121d)

b. que viinham todos armados de armas brãcas (CroGer, 126c)

c. ficarom todos spantados (CroGer, 126d) 
d. ainda que hy fossem os reynos mesturados. (CroGer, 127c)

e. que ante nom fossem todos mortos (CroGer, 128b)

(18) a. Ve'spera de Pinticoste foi grande gente assũada em Camaalot (Demanda, 1a)

b. Deus mande que seja a cavalaria tam bem empregada em vo's como em nosso linhagem. (Demanda, 2c)

c. ca hoje sera'a minha corte chamada por sempre Corte Aventurosa (Demanda, 5a)

d. bem seja el viindo (Demanda, 7d)

e. $\quad$ E tanto que entrou $\mathrm{i}$ foi o paaço todo comprido de bõõ odor (Demanda, 8a)

porque fora todo mui bem tratado e encaminhado (Zurara, 2)

(20) a. depois que ouve os seus todos ajuntados (CroGer, 118b)

b. disserõ que lhes semelhava que avyam Deus yrado (CroGer, 119c)

c. E diziã que tiinhã grande torto recebudo dos Castellããos (CroGer, 121c)

d. que seja per ti toda Castella defendida (CroGer, 122c)

e. Ca Almançor tẽ ora o moor poder ajuntado que elle nũca teve (CroGer, 123b)

f. E levava a boca aberta e lançava per ella chamas de fogo (CroGer, 124b)

g. que nõ leixasse Castella dessemparada em aquelle dya. (CroGer, 125a)

(21) a. que visse a Ta'vola Redonda comprida (Demanda, 5a)

b. quando vejo a seeda perigosa comprida (Demanda, 5c)

c. saibas que ha's tu nome britado dês hoje a manhãã (Demanda, 6c)

(22) a. Hũa monja nobre de linhagem e de grande santidade foi assi pollo imiigo enguanada (CastPer, 3r)

b. $\quad$ ca $\mathrm{D}(\mathrm{eu}) \mathrm{s}$ tem semp(re) os braç(os) abert(os) p(er)a rreceb(e)r os pecador(e)s a misericórdia (CastPer, 8v)

(23) a. sey que lealmente he todo scripto quanto meu pequeno saber, embargado em todo geeral regimento de justiça, consselhos e todas outras proveenças de meus Reynos e Senhorio, pode percalçar (LeaCon, 3r)

b. $\quad$ E por que ao presente de sua mercee tem esta virtude outorgada em estes Reynos antre senhores e servidores, maridos e molheres, tam perfeitamente que (LeaCon, 3v)

La interpolación de constituyentes también se documenta en las construcciones de infinitivo recogidas en los textos portugueses analizados. En total se han registrado 156 casos en los que algún constituyente aparece entre el verbo regente y el infinitivo complemento. Esta cifra forma el 18,7\% de las 835 ocurrencias de construcciones de infinitivo. Si comparamos la proporción de este fenómeno en los textos medievales es decir, anteriores al siglo XV-y en los renacentistas -del siglo XV-, no podemos 
encontrar diferencias considerables: en los primeros la frecuencia de la interpolación es del 16,1\% -92 casos de los 571 ejemplos medievales-, mientras que en los últimos alcanza el 17,6\% -64 casos de los 364 ejemplos renacentistas-. Estos datos se observan en el Cuadro 3.5.

\begin{tabular}{|c|c|c|c|c|c|c|}
\hline Textos & \multicolumn{4}{|c|}{ Int+ } & Int- & Total \\
\hline & Sujeto & Objeto & Adverbio & Int + total & & \\
\hline ForRea & 2 & 2 & 2 & 6 & 88 & 94 \\
\hline CroGer & 12 & 19 & 23 & 54 & 267 & 321 \\
\hline Demanda & 14 & 7 & 11 & 32 & 124 & 156 \\
\hline Zurara & 2 & 3 & 10 & 15 & 80 & 95 \\
\hline CasPer & 19 & 2 & 13 & 34 & 145 & 179 \\
\hline LeaCon & 1 & 4 & 10 & 15 & 75 & 90 \\
\hline Total & 50 & 37 & 69 & 156 & 779 & 835 \\
\hline
\end{tabular}

La proporción de la intercalación con infinitivo en portugués

Al comparar los datos de frecuencia de cada uno de los textos también podemos observar un equilibrio general: en la mayoría de los textos encontramos una proporción oscilante entre el $15,8 \%$ y el $20,5 \%$, y únicamente en el texto legal se registra un porcentaje notablemente bajo. Los datos concretos según textos son los siguientes: ForRea: 6,4\%; CroGer: 16,8\%, Demanda: 20,5\%; Zurara: 15,8\%; CasPer: 19\%; LeaCon: $16,7 \%$.

Por lo que se refiere a la categoría de los constituyentes intercalados los datos son compatibles con los que observamos en español: los elementos más veces intercalados son los complementos circunstanciales, los cuales aparecen interpolados 69 veces, es decir, en el 8,3\% de los 835 casos de construcciones de infinitivo; en (24-29) se aducen aquellos ejemplos recogidos durante la investigación que contienen elementos adverbiales intercalados.

(24) a. nõ possa d(e)poys deytarllo p(er) nenhua destas razoes (ForRea, 79r)

b. $\quad$ E se nõ quiserẽ end(e) auijr os omees boos que recebã proua da suspeyta (ForRea, 78)

(25) a. Agora leixaremos aquy de fallar desta razom (CroGer, 114a)

b. $\quad$ E os Esturããos ẽvyarom entom enganosamente dizer a el rey dom Ramiro que (CroGer, 114c)

c. e começou logo a pobrar, enno reyno deste rey dom Ramiro, Sallamãca e Ledesma, Ribas e Banhos e a Alfandega e Pena Gussende e outros muytos castellos; (CroGer, 115b)

d. e foy a terra de mouros correr hũũ castello que antigamente avya nome hy Auguas e he aquelle a que hora dizem Tallaveira (CroGer, 116a)

e. [E desi leixou ante todos de reinar logo e desemparousse do reino que lhe dera seu irmãão dom Afonso] (CroGer, 116a) 
f. $\quad$ e veeo enton fazer pazes cõ Abdenaamer (CroGer, 117b)

g. Agora leixaremos aquy de fallar [del rei dom Sancho] (CroGer, 117c)

h. e nõ podia ja husar [d]'armas como comprya (CroGer, 117d)

i. E elle mandou logo desenvolver o seu pendom [e] (CroGer, 119d)

j. $\quad$ E o conde mandou logo chamar todos seus altos homẽẽs: (CroGer, 120c)

k. e ferirõnos assy fortemẽte que lhes fezerõ per força leixar o campo (CroGer, 121b)

1. E, empero era ainda mal treyto daquella lançada que lhe el rey de Navarra dera, nõ leixou porẽ dhyr contra o cõde de Tolosa e cõtra os Navarros (CroGer, 121c)

m. ca te nõ quero mais dizer (CroGer, 123a)

n. que vos nõ posso ora dizer (CroGer, 123b)

o. $\quad$ Ca me fazes sẽpre andar ẽ coyta e ẽ mãrteiro (CroGer, 123c)

p. que erã muy boos mancebos e muy de prol que [d]everamos cõ os primeiros [contar] (CroGer, 123d)

q. $\quad$ que se os nõ podessem ẽ esse dya vẽcer (CroGer, 124a)

r. virom essa noyte vĩur voando pello aar hũũ dragõ muy grande e muy spantoso (CroGer, 124b)

s. ca acharom muytos mouros no camynho de que se ouverõ ante de livrar (CroGer, 124d)

t. e queria ja escurecer (CroGer, 125b)

u. $\quad$ que nõ podya mais seer (CroGer, 126b)

v. ca lhes poderia dello crecer dampno. (CroGer, 126d)

w. e ouvira ja dizer delle tãtas bondades e outrossy do açor (CroGer, 127b)

(26) a. feze-os mui bem servir (Demanda, 1a)

b. nem el nom no podia ja mais cuidar que (Demanda, 2b)

c. $\quad$ E el-rei fora entam ouvir missa aa see (Demanda, 2d)

d. virom i seer ũũ cavaleiro (Demanda, $3 b$ )

e. que vi assi nadar por sobre a a'gua como se fosse madeiro (3c)

f. Maravilha pode ende aviür (Demanda, 5d)

g. que quiserom mais saber dela (Demanda, 6c)

h. de guisa que muitos veri'ades i cair (Demanda, 7a)

i. $\quad$ bem ouvira ja' dizer que (Demanda, 7d)

j. $\quad$ u os outros nom poderom i aviĩr (Demanda 2a)

k. $\quad$ que me leixaste tanto viver que (Demanda 5a)

(27) a. e os fez nobremente agasalhar (Zurara, 1)

b. Qual cousa poderemos mais razoadamente desejar que a paz? (Zurara, 4)

c. que sem ela nenhũa cousa, per grande e boa que seja, nam se pode em seu perfeito estado conservar (Zurara, 4)

d. Quem será aquele que possa em este mundo fazer cousa, por santa e bõa que seja, que (Zurara, 7)

e. Quem poderia aaquele tempo falar em outra cousa senam (Zurara, 9) 
g. Nom era porém alguũ que podesse certamente, nem assi apalpando, falar na cidade de Cepta. (Zurara, 15)

h. Quisera, meus filhos - disse ela contra os Ifantes- dar-vos agora as espadas em que vos ante falei (Zurara, 18)

i. $\quad$ E estas espadas mandei assi fazer pera as dar a vós (Zurara, 19)

j. $\quad$ Nom poderia bem declarar per escrito a grande tristeza com que o Ifante D. Anrique estava (Zurara, 21)

(28) a. diz elle que quem se quer sajement(e) confessar e achar graça ant(e) $\mathrm{D}$ (eu)s (CasPer, 2r)

b. que saibha bem conhecer o pecado e consselhar o pecador (CasPer, 2r)

c. que podemos hi filhar enxenplo. (CasPer, 3r)

d. quem se bem $q(u e) r$ confesar deve com gram dilige^ncia penssar em todos os malles que fez (CasPer, $4 \mathrm{r}$ )

e. que sem a ajuda de $\mathrm{D}(\mathrm{eu}) \mathrm{s}$ nom podem(os) bem faz(er) (CasPer, 5r)

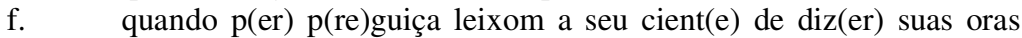
canônicas (CasPer, 6r)

g. $\quad \mathrm{p}(\mathrm{er})$ que homem pode clarament(e) conhecer sua maa voontade (CasPer, 9r)

h. E ella os fez logo thirar (CasPer, 10r)

i. deve-se triguosament(e) (con)fessar (CasPer, 10v)

j. $\quad$ deve deligentemente buscar toda sua conciência (CasPer, 10v)

k. $\quad$ E ainda por ap(re)nder bem a sse confessar q(ue) (CasPer, 14r)

1. $\mathrm{q}(\mathrm{ue})$ homem possa seguramente edeficar seu castello (CasPer, 14v)

m. que o nom possa bem thirar (CasPer, 15v)

(29) a. Podelloees, se vos praz, chamar leal consselheiro (LeaCon, 3v)

b. Compre, pera sse melhor entender, de se leer todo de começo (LeaCon, 3v)

c. por que algũas cousas se podem bem razoar que nom som taaes pera screver (LeaCon, 3r)

d. costumandonos a novamente aprender aquellas cousas que pera o estado em que formos perteecerem (LeaCon, $4 \mathrm{v}$ )

e. per que se demostra como devemos sempre teer esta teençom (LeaCon, 5r)

f. $\quad$ E aquesta parte se requere nom se trigar nas determinaçõoẽs das cousas (LeaCon, 5r)

g. por que aquella nom conssente em tal guisa contradizer as duas primeiras que (LeaCon, 7r)

h. Quarta, que desejemos muyto percalçar e aver todas estas partes do entendimento, (LeaCon, 5r)

i. a qual lembrança me parece principalmente aa cabeça perteencer (LeaCon, 6r)

j. mas querendo sem descliçom comprir quanto esta voontade spiritual demanda, (LeaCon, 7v) 
El sujeto se registra interpolado 50 veces, o sea, en el $6 \%$ de todos los casos de construcciones de infinitivo. Nótese que este orden no es imposible en el portugués actual, aunque su uso tampoco es natural. Los casos con sujeto intercalado se presentan en (30-35).

(30) a. se nõ for daquelles que manda o foro receb(er) sẽ p(es)soarya (ForRea, 78r)

b. $\quad$ Estas sõ as razoes p(er) que podẽ os alcaydes seer deytados por susp(e)ytos dos p(re)ytos q(ue) nõ iuygẽ: (ForRea, 78v)

(31) a. per que o podesse elle aver (CroGer, 116b)

b. $\quad$ E ẽtom mandou o conde dom Fernã Gonçallvez fazer hũũ ataude muy bõõ e muy rico (CroGer, 121d)

c. nõ quedava elle de dar muy grãdes golpes (CroGer, 125a)

d. E, dally adyãte, começou o conde a manteer sua terra mais seguramente que ante fazia (CroGer, 117b)

e. Aqui leixa a estorya de fallar destas razõões (CroGer, 117c)

f. E entõ mãdou / logo o conde mover sua hoste (CroGer, 120d-121a)

g. $\quad$ Ora leixa o conto de fallar desto (CroGer, 121d)

h. $\quad$ ca, per nẽ hũa outra maneira que eu fezesse, nõ poderya eu vencer tã grandes poderes (CroGer, 122c)

i. que o nõ poderia homẽ pẽsar (CroGer, 122c)

j. $\quad$ E entõ ouverõ todollo seus de ajuntar ally onde estava a sua syna (CroGer, 125b)

k. $\quad$ per que possa eu seer livre desta prema dos Leoneses! (CroGer, 127b)

1. que te nõ poderia eu gradecer cõ serviço (CroGer, 125a)

(32) a. assi que podera homem i veer mui gram gente, (Demanda, 1a)

b. queredes vo's seer cavaleiro? (Demanda, 1c)

c. Entam começarom todos a chorar com prazer (Demanda, 1d)

d. Esto quero eu milhor saber por veer as grandes aventuras e milagres que Deos por ti fara'. (Demanda, 2b)

e. ca, em aquel tempo, nom podia homem achar em todo o regno de Logres donzel tam fremoso nem tam bem feito (Demanda, 2b)

f. era tal que nom podia homem achar rem em que lhe travasse (Demanda, 2b)

g. ca me quero eu ir aa corte (Demanda, 2c)

h. que, tanto que aventura vos veesse, nom soi'ades vo's a comer em niũa gram festa (Demanda, 3a)

i. que em esta corte de sua idade nom devia homem mais preçar homem mancebo de cavalaria (Demanda, 4c)

j. mandou el-ei cobrir as mesas (Demanda, 4d)

k. ca por al nom fiz eu viir tanta gente aa minha corte senam (Demanda, 5a)

1. $\quad$ esto podes tu bem veer (Demanda, 6c)

m. Ca a nom quer nĩũ provar (Demanda, $6 \mathrm{c}$ ) 
n. que nunca tam altamente começara cavaleiro a dirribar cavaleiros (Demanda, 7b)

(33) a. Boa espada, que, quando Deos queria, soíees vós a cortar per cima das solhas e das cotas (Zurara, 24)

b. Nom vos disse eu que ante de amanhaã avia meu filho de seer de todo prestes? (Zurara, 27)

(34) a. A esta paz nom pode algũu vĩ̃r se nom ha' v(er)dadeira cont(ri)çom e door no coraçom (CasPer, 1v)

b. Por isto nom deve homem leixar confessar todollos pensament(os) que trazem pecado (CasPer, 3r)

c. E deve-sse homem a nembrar de se(us) pecad(os) com grande door do coraçom (CasPer, 4r)

d. que em hũa soo ora pode homem guaanhar o parai'so. (CasPer, 6v)

e. $\quad$ assi deve homem peenssar, se (CasPer, 7v)

f. deve-sse homem a confessar (CasPer, 9v)

g. E isto deve homem a fazer nom por vãa gllória, nem por louvor (CasPer, 11v)

h. Deve homem $\operatorname{diz}(\mathrm{er})$ as circonstâncias que agravom o pecado (CasPer, 12r)

i. $\quad$ Assi deve homem peensar se (CasPer, 13r)

j. $\quad$ Por isto deve homem mostrar que lhe nom praz (CasPer, 13r)

k. Mes os penssament(os) em que ha' delleito, deve homem a dizer quanto lhe deu de conssintimento e o tempo que em elles tardou (CasPer, 13r)

1. Assi se deve homem a confessar do aficado cuidado que teve (CasPer, 13r)

m. assi deve homem de rrecobrar e comp(ri)r o que fez menos do que comp(ri)a (CasPer, 14r)

n. $\quad \mathrm{P}[\mathrm{o}]$ llo pecado do poboo, leixo eu rreinar o ipo'cc(ri)ta (CasPer, 15r)

o. deve o bõo rrelligioso teer em gram cortesia (CasPer, 15r)

p. Isto deve homem fazer por $\mathrm{D}(\mathrm{eu}) \mathrm{s}$ purament(e) (CasPer, 15v)

q. E por isto nom deve homem mete(r) seu coraçom tanto em algũa creatura que (CasPer, 15v)

r. $\quad \mathrm{O}$ amor d'outrem nom pode algũu melhor aver que p(er) amar (CasPer, 15v)

s. Bem pode homem sagement(e) e sem queixume esquiva(r) a companhia daquell[e]s que (CasPer, $15 \mathrm{v}$ )

por que ante da viinda de nosso senhor ja mandavam os homẽẽs apousentar de \#LXX ãnos (LeaCon, 5v)

Se han registrado 37 casos en el corpus cuando el elemento que separa el verbo regente del infinitivo es algún complemento regido -argumento interno- de alguno de los verbos. Esta cantidad de ocurrencias constituye el el 4,4\% de los 835 casos con infinitivo. Nótese que entre los complementos regidos se han tenido en cuenta 
también los agentes de los infinitivos que en la superficie aparecen como complementos de un verbo causativo o de un verbo de percepción -como ver en (37b) o (38a), por ejemplo-. Recuérdese que la intercalación de complementos regidos por el infinitivo es completamente inaceptable en el portugués actual. Los casos que contienen complementos regidos intercalados entre los dos verbos de la construcción se presentan en (36-41).

(36) a. E cada huu se guarde d(e) mal faz(er) (ForRea, 72r)

b. $\quad$ se aquel a q(ue) fazã reuolta poder esto prouar (ForRea, 78v)

(37) a. e vyo o porco estar ?de?tras [do altar] (CroGer, 118d)

b. quando o conde vyo o porco hir per aquella pena açima (CroGer, 118d)

c. Como os Navarros forõ ao cõde Piteus e ao conde de Tollosa querellarlhes a morte del d. rey dom Sancho (CroGer, 121b)

e. aveolhe que vyu o conde Peytavinos de Tollosa ãdar / pella lide embaratandosse cõ os seus (CroGer, 121c-d)

f. e mãdou ẽ elle meter o corpo do conde de Tollosa (CroGer, 121d) (O)

g. E que outrossi mãdara alfajares pellas terras de mais lõge a preegar esto medês, (CroGer, 122a)

h. $\quad$ ẽtrey na igreja a fazer mynha oraçõ (CroGer, 123a)

i. $\quad$ ca eu fuy a hũa hermyda veer hũũ meu amigo (CroGer, 123a)

j. $\quad$ que os nõ poderyã melhores achar em nẽũ hũa parte (CroGer, 124a)

k. que fezerõ ally vĩir aquella semelhança tal pera lhes poer spanto (CroGer, 124b)

1. E elles lhe disserom ẽ como virõ o dragõ vĩur voando pello aar (CroGer, 124b)

m. E, per esta maneira, fezerõ aquy vĩ̃r esta besta pera nos poer medo e espanto (CroGer, 124b)

n. que nẽ hũũs outros homẽẽs o nõ poderyã melhor fazer (CroGer, 126a)

o. $\quad$ que nõ podya melhor seer (CroGer, 127b)

p. $\quad$ que se nõ podem no mundo melhores saber (CroGer, 127b)

q. que virom seus senhores estar em tal perigoo (CroGer, 128b)

r. $\quad$ quãdo o conde vyu el rey assi vĩur (CroGer, 128b)

s. $\quad$ que nom podya hy al fazer (CroGer, 128b)

(38) a. quando el viu Lançarot ir (Demanda, 1b)

b. E por em quero todos teus feitos saber que acabara's, (Demanda, 2a)

c. que nom podia de maior seer (Demanda, 2b)

d. ca hora de terça hei i de seer (Demanda,2c)

e. nom se podia a el chegar niũũ que (Demanda, 3b)

f. nom podiam rem dizer (Demanda, 5b)

g. que quisestes aqui vĩur (Demanda,6b)

(39) a. E nom podiam em al falar (Zurara, 3)

b. $\quad$ E des i meteram suas mãos a revolver as armas (Zurara, 24)

c. pera ajudarem el-rei seu primo a conquistar o reino de França (Zurara, 12) 
(40) a. q(ue) ant(e) leixariom o que ham apodrecer, que darem a se(us) irmãaos ou a se(us) s(er)vidor(e)s (CasPer, 7v)

b. devem-se d(e)lle a confessar (CasPer, 7v)

(41) a. querendo todo melhor declarar (LeaCon, 3r)

b. querendo a algũũs aproveitar e a nenguem empeecer (LeaCon, 4r)

c. Porem bem sey que algũa leitura nom pode a todos igualmente prazer (LeaCon, 4r)

d. a qual, por querer ambas satisfazer, sem nem hũũ agravamento põoẽ o que (LeaCon, 6v)

\section{Resumen y conclusiones}

En las páginas precedentes se han presentado los datos de frecuencia referentes a la anteposición del participio y del infinitivo al verbo que los rige y a la interpolación de constituyentes entre el verbo regente y el participio o el infinitivo complementos. El estudio paralelo de los dos tipos de construcciones confirma los datos ofrecidos por trabajos precedentes sobre las construcciones de participio del español medieval y, al mismo tiempo, sobre el caso de las construcciones de infinitivo del español presentados en este mismo tomo. Los datos de frecuencia de los dos fenómenos examinados son parecidos en el caso de ambos tipos de construcciones: la proporción de la anteposición con participio parece subir allí donde sube también con infinitivo, aunque con éste último es algo inferior; la interpolación se mantiene por lo general al mismo nivel en ambos tipos de construcciones. En cuanto a la proporción de las ocurrencias de los dos fenómenos en los textos medievales y en los renacentistas, podemos observar que el porcentaje de la anteposición es inferior en los textos del siglo XV, excepto LeaCon, mientras el de la interpolación no cambia considerablemente. La comparación de los datos según textos también indica detalles interesantes. De un lado, podemos observar que entre todos los textos hay uno, en concreto el texto legal medieval -ForRea-, en el cual ambos fenómenos considerados arcaizantes alcanzan una frecuencia muy baja. De otro lado, entre los textos del siglo XV también hay uno -a saber, LeaCon-, en el que los mismos fenómenos tienen un porcentaje relativamente alto. Estos datos sugieren que el uso de los fenómenos en cuestión, entendidos como alternativas sintácticas arcaizantes, caracterizaba más bien los textos de más valor estilístico, y más complejos desde el punto de vista discursivo -vid. supra-; desde este punto de vista es lógico que ForRea, texto legal, presente sólo unos cuantos casos de estas variantes, frente a los textos narrativos como CroGer y Demanda. La frecuencia baja de la anteposición en Zurara y CasPer en el siglo XV indica, probablemente, que tal arcaísmo no caracterizaba ya la lengua de esta época, por lo menos a los autores de estilo más sencillo. El porcentaje más alto del mismo fenómeno en LeaCon puede ser atribuido, quizás, a la influencia del estilo latinizante, frecuente entre los autores cultos de la época del humanismo. 